\title{
VALIDAÇÃO DAS DEFINIÇÕES DE TERMOS IDENTIFICADOS NO PROJETO CIPESC PARA O EIXO FOCO DA PRÁTICA DE ENFERMAGEM DA CIPE ${ }^{\circledR}$
}

\author{
VALIDATION OF THE DEFINITION OF THE TERMS IDENTIFIED IN THE CIPESC \\ PROJECT FOR THE AXIS FOCUS OF NURSING PRACTICE OF ICNP ${ }^{\circledR}$
}

\author{
VALIDAD DE LAS DEFINICIONES DE TÉRMINOS IDENTIFICADOS EN EL PROYECTO \\ CIPESC PARA EL EJE FOCO DE LA PRÁCTICA DE ENFERMERÍA DE CIPE ${ }^{\circledR}$
}

\author{
Telma Ribeiro Garcia ${ }^{1}$ \\ Maria Miriam Lima da Nóbrega² \\ Maria Clemilde Mouta de Sousa ${ }^{3}$
}

\begin{abstract}
RESUMO: Em continuidade à contribuição da Enfermagem brasileira para a elaboração da CIPEâ, que está sendo construida sob a coordenação do CIE, objetivou-se neste trabalho definir os termos identificados no projeto CIPESC e classificados no eixo foco da prática de enfermagem da Classificação de Fenômenos de Enfermagem da CIPE ${ }^{\circledR}$ - versão Beta e validar essas definições junto a um grupo de juizes composto por profissionais com envolvimento anterior na execução do projeto CIPESC, ou com envolvimento docente ou assistencial na área de Enfermagem em Saúde Coletiva. O processo foi realizado em duas fases, considerando-se validadas as definições que alcançaram um Índice de Concordância $(\mathrm{IC})^{3} 0,80$ entre o grupo de juizes. Noventa e nove definições de termos foram validadas: 26 alcançaram IC igual a 1,00; 56 alcançaram IC entre 0,90 e 0,99 e 17 alcançaram IC entre 0,80 e 0,89. A definição de um termo não foi validada.

PALAVRAS-CHAVE: linguagem especial, sistema de classificação, fenômeno de enfermagem, saúde coletiva
\end{abstract}

ABSTRACT: This work aims at continuing the contribution of Brazilian nursing in the elaboration of ICNPâ, which is being constructed by the coordination of ICN. It has two main objectives which are: 1) the definition of the terms identified in the CIPESC project and classified in the axis focus of nursing practice of the Nursing Phenomena Classification of ICNPâ- Beta Version; 2) the validation of these definitions within a group of judges composed of professionals who were previously involved in the execution of the CIPESC project, or who were teachers or nurse assistants in the area of community health nursing. The process was carried out in two phases, considering that the definitions that had $a^{3} 0.80$ rate of Agreement (RA) within the group of judges were validated. Ninety-nine definitions of the terms were validated: 26 attained a RA equal to 1.00; 56 attained a RA between $0.90-0.99$ and, 17 attained a RA between 0.80 an 0.89 . The definition of one term was not validated.

KEYWORDS: special language, classification system, nursing phenomenon, collective health

RESUMEN: En continuidad a la contribución de la Enfermería brasileña para elaborar la CIPEâ, que se está construyendo bajo la coordinación del CIE, se busca en este trabajo definir los términos identificados en el proyecto CIPESC y clasificados en el eje foco de la práctica de enfermería de la Clasificación de Fenómenos de Enfermería de CIPEâ - versión Beta y validar esas definiciones junto a un grupo de jueces, que está compuesto por profesionales relacionados con la ejecución del proyecto CIPESC, o que tengan afinidad con la docencia o asistencia dentro del área de Enfermería en Salud Colectiva. La investigación se realizó en dos fases, y se consideraron validadas las definiciones que alcanzaron un Índice de Concordancia (IC) $>0,8$ entre el grupo de jueces. Noventa y nueve definiciones de términos se validaron: 26 alcanzaron IC igual a 1,00; 56 alcanzaron IC entre 0,90 y 0,99 y 17 alcanzaron IC entre 0,80 y 0,89. La definición de un término no se validó.

PALABRAS CLAVE: Ienguaje especial, sistema de clasificación, fenómeno de enfermeria, salud colectiva

Recebido em 03/01/2001

Aprovado em 06/02/2002

\footnotetext{
1 Enfermeira, Doutora em Enfermagem pela EERP-USP. Professora do Departamento de Enfermagem de Saúde Pública e Psiquiatria, Centro de Ciências da Saúde - UFP.

${ }^{2}$ Enfermeira, Doutora em Enfermagem pela UNIFESP. Professora do Departamento de Enfermagem de Saúde Pública e Psiquiatria, Centro de Ciências da Saúde - UFP.

${ }^{3}$ Enfermeira, Mestre em Enfermagem pela UFPB. Doutoranda em Enfermagem na UFC. Professora do Departamento de Enfermagem de Saúde Pública e Psiquiatria, Centro de Ciências da Saúde - UFP.
} 


\section{INTRODUÇÃO}

Em 1991, o Conselho Internacional de Enfermeiras (CIE) iniciou a elaboração e implementação da Classificação Internacional para a Prática de Enfermagem (CIPE $\left.{ }^{\circledR}\right)$, que representa um marco unificador de todos os sistemas de classificação dos elementos da prática de enfermagem (diagnósticos, intervenções e resultados de enfermagem) disponiveis na área, no ââmbito mundial. No processo de construção da $\operatorname{CIPE}^{\circledR}$, percebeu-se que, embora alguns dos sistemas de classificação existentes já incorporassem termos relacionados a esses campos da prática, ainda havia a necessidade de se identificar e incluir novos termos associados à atenção primária e à prática de enfermagem em serviços comunitários de saúde.

Partindo dessa constatação, o CIE constituiu um grupo de trabalho especialmente designado para participar de um projeto que objetivava elaborar e divulgar processos para identificação de termos que pudessem ser usados pelas enfermeiras de qualquer pais para evidenciar a linguagem de enfermagem em cuidados primários e em serviços comunitários de saúde; identificar e garantir a incorporação desses termos na CIPE ${ }^{\circledR}$; estabelecer mecanismos no âmbito dos países participantes que possibilitassem, após o término do projeto, a continuidade de contribuição para a $\mathrm{CIPE}^{\circledR}$, com termos relacionados a cuidados primários e a serviços comunitários de saúde; aumentar o reconhecimento, por parte dos profissionais e das autoridades de saúde dos paises envolvidos, da necessidade de incluir dados relacionados à prática de enfermagem nos sistemas de informação em saúde (ICN, 2000).

A participação da Enfermagem brasileira nesse esforço internacional aconteceu a partir de 1994, sendo esse um compromisso assumido pela ABEn Nacional, que delineou e executou o Projeto de Classificação da Prática de Enfermagem em Saúde Coletiva no Brasil (CIPESC), com o qual se intentava revelar a dimensão, a diversidade e a amplitude das práticas de enfermagem no contexto do Sistema Único de Saúde (ABEn, 1996; EGRY; MISHIMA; ANTUNES, 1999).

Tendo em vista a dimensão continental do pais, a sua diversidade sócio-econômica e cultural e o acesso geográfico, foram definidos 15 cenários de pesquisa, representantes de todas as regiões do pais, para a operacionalização das atividades do projeto CIPESC. Em uma primeira fase, foram coletados dados para caracterização dos 15 cenários e da força de trabalho de enfermagem, dados esses submetidos a uma análise quantitativa e editados pela $A B E n$ Nacional na publicação "A Classificação Internacional das Práticas de Enfermagem em Saúde Coletiva"
(CHIANCA; ANTUNES, 1999). Em seguida, para a coleta de dados acerca das práticas dos trabalhadores envolvidos no processo de trabalho de enfermagem em saúde coletiva, foram empregadas as técnicas de grupo focal e entrevista semi-estruturada. Os dados coletados nessa segunda fase da pesquisa foram submetidos a uma análise qualitativa e resultaram em duas vertentes de resultados: 1) a produção de um inventário vocabular de enfermagem em saúde coletiva, a partir da identificação de termos relacionados a fenômenos e a ações de enfermagem; e 2) a caracterização do processo de trabalho de enfermagem em saúde coletiva $\mathrm{n}$ o Brasil. Os resultados gerais do projeto CIPESC estão editados na publicação "Sistema de Classificação da Prática de Enfermagem: um trabalho coletivo" (GARCIA; NÓBREGA, 2000a).

A primeira vertente de resultados qualitativos "Inventário Vocabular de Enfermagem em Saúde Coletiva" - propunha-se a identificar os termos empregados no Brasil, no campo da saúde coletiva, que caracterizam a linguagem especial da enfermagem; realizar uma análise dos termos identificados, a partir dos campos semânticos de fenômenos e ações de enfermagem propostos na CIPE ${ }^{\circledR}$ - versão Beta; comparar os resultados obtidos com os sistemas de classificação de fenômenos e ações de enfermagem da CIPE ${ }^{\circledR}$ - versão Beta, para identificação de termos incluídos e não incluídos, que pudessem expandir a CIPE ${ }^{\circledR}$ (GARCIA; NÓBREGA, 2000b). Na elaboração desse inventário vocabular, foram identificados 331 termos no eixo foco da prática de enfermagem da Classificação de Fenômenos de Enfermagem da CIPE ${ }^{\circledR}$ - versão Beta, dos quais 152 eram termos já incluídos e 179 eram termos não incluídos nessa classificação.

Como uma continuidade do esforço de contribuição da Enfermagem brasileira para a construção da CIPE ${ }^{\circledR}$, verificou-se a necessidade de definir os termos identificados no projeto CIPESC e que ainda não estavam incluidos no eixo "foco da prática de enfermagem da Classificação de Fenômenos de Enfermagem" da CIPE ${ }^{\circledR}$ - versão Beta e, posteriormente, de validar as de finições elaboradas, ou seja, de confirmar se coincidem ou diferem do significado atribuido a esses termos por pessoas que exercem atividades profissionais na área de Enfermagem em Saúde Coletiva. Foram esses os objetivos deste trabalho.

\section{METODOLOGIA}

Conforme relato de Garcia e Nóbrega (2000b), durante a segunda fase de coleta de dados do projeto CIPESC, foram realizadas, nos 15 diferentes cenários de pesquisa, 90 reuniões utilizando-se a técnica de 
grupos focais, envolvendo a participação de 720 componentes da equipe de enfermagem - enfermeiros, técnicos de enfermagem, auxiliares de enfermagem e agentes comunitários; e 165 entrevistas individuais com gerentes de unidades básicas, gestores e representantes de entidades ligadas à saúde e à enfermagem.

Para a realização do "Inventário Vocabular de Enfermagem em Saúde Coletiva", trabalhou-se com os dados transcritos de 49 reuniões de grupos focais (28 de enfermeiras, 20 de técnicos e auxiliares de enfermagem e 1 de agentes comunitários de saúde), realizadas em 14 dos cenários do projeto. Constituíram o corpus de análise dessa vertente de resultados as unidades de análise recortadas dos dados transcritos, consideradas ad hoc como pertinentes para os objetivos estabelecidos, a saber, termos ou frases que indicassem os fenômenos e as ações que constituem a linguagem especial de enfermagem no campo da saúde coletiva (GARCIA; NÓBREGA, 2000b).

Recortadas as unidades de análise, foram realizadas as seguintes fases: elaboração de uma "listagem bruta" de fenômenos e ações de enfermagem, por componente da força de trabalho; elaboração, ainda por componente da força de trabalho, da listagem de fenômenos e ações em ordem alfabética, para identificação e retirada das repetições; elaboração de uma listagem única (não mais por componentes da força de trabalho) de fenômenos e ações de enfermagem, com nova retirada de repetições; classificação dos termos relacionados a fenômenos de enfermagem segundo os oito eixos propostos na Classificação de Fenômenos de Enfermagem da CIPE ${ }^{\circledR}$ - versão Beta (foco da prática de enfermagem, julgamento, freqüência, duração, topologia, lugar do corpo, probabilidade e portador) e classificação preliminar dos termos ou frases relacionados a ações de enfermagem segundo o eixo tipo de ação da Classificação de Ações de Enfermagem da CIPE ${ }^{\circledR}$ - versão Beta (GARCIA; NÓBREGA, 2000b).

No eixo foco da prática de enfermagem da Classificação de Fenômenos de Enfermagem, conforme já afirmado anteriormente, foram identificados 179 novos termos com potencial para inclusão na CIPE ${ }^{\circledR}$. Durante o processo de definição de termos realizado neste trabalho, esse número ficou reduzido a 100, haja vista que o significado de 79 deles era igual ou semelhante ao de termos já incluidos na CIPE ${ }^{\circledast}$ - versão Beta. Para a definição de alguns desses 100 termos utilizou-se dicionários e a literatura disponivel, nem sempre atualizada ou especifica da área; para outros, a definição apresentada resultou do entendimento das autoras do trabalho, não tendo sido identificada literatura que respaldasse esse entendimento.

Elaboradas as definições, construiu-se um instrumento contendo, além de instruções para preenchimento, os 100 termos e respectivas definições. Esse instrumento foi enviado a um grupo de juizes composto por enfermeiras(os) com envolvimento anterior na execução do projeto CIPESC, ou com envolvimento docente ou assistencial na área de Enfermagem em Saúde Coletiva, para que opinassem acerca das definições.

Em respeito ao previsto na Resolução 196/96 (BRASIL, 1996), ressaltou-se na parte introdutória do instrumento que a participação era voluntária; que, na divulgação dos resultados, seria garantido o anonimato das(os) respondentes e que a devolução do instrumento devidamente preenchido seria compreendida como aquiescência para participar do processo.

Quanto à validação das definições, solicitouse que, após a leitura da definição de cada um dos 100 termos listados no instrumento, a(o) respondente apresentasse sua opinião, marcando se concordava ou se discordava dela. Em caso de discord®ncia, pediu-se que $\mathrm{a}(\mathrm{o})$ respondente sugerisse a alteração necessária, indicando também, se fosse o caso, a referência bibliográfica que respaldava a sugestão. Considerou-se como estando validada toda definição que alcançasse um Índice de Concordância (IC) entre juizes ${ }^{3} 0,80$, calculado através da fórmula $I C=N C$, $\mathrm{NC}+\mathrm{ND}$, em que $\mathrm{NC}=$ número de concord®ncias e $\mathrm{ND}=$ número de discordâncias (BATISTA, 1984).

O processo de validação foi realizado em duas fases subseqüentes. Na primeira fase, de que participaram 37 juizes, foram validadas as definições de 54 dos 100 termos constantes do instrumento. Para a segunda fase, re-elaborou-se o instrumento, excluindo-se os termos cujas definições já haviam sido consideradas validadas e incorporando-se as sugestões das(os) respondentes para as definições dos 46 termos que não alcançaram o IC desejado na primeira fase de validação. Esse segundo instrumento foi enviado para o mesmo grupo que havia participado da fase anterior, recebendo-se, em retorno, 17 deles devidamente preenchidos. Na segunda fase, foram validadas as definições de 45 termos.

\section{RESULTADOS}

No processo de validação das definições dos termos identificados no projeto CIPESC e classificados no eixo foco da prática de enfermagem da Classificação de Fenômenos de Enfermagem da CIPE ${ }^{\circledR}$ - versão Beta, 26 alcançaram IC igual a 1,00; 56 alcançaram IC entre 0,90 e 0,99; 17 alcançaram IC entre 0,80 e 0,89 e a definição de um termo não alcançou $\mathrm{IC}^{3} 0,80$ nem na primeira, nem na segunda fase de validação. 
GRÁFICO 1 - Distribuição de freqüência das definições dos termos identificados no projeto CIPESC e classificados no eixo foco da prática de enfermagem da Classificação de Fenômenos de Enfermagem da CIPE ${ }^{\circledR}$ versão Beta, segundo Índice de Concordância (IC) alcançado no processo de validação

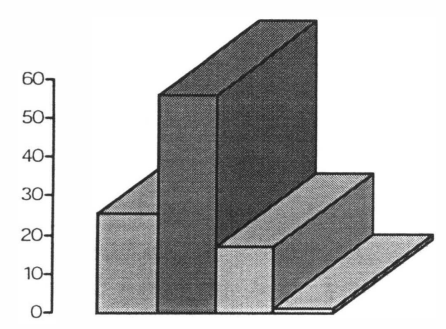

A seguir, serão apresentados, em dois quadros distintos, segundo fase de validação, os termos identificados no projeto CIPESC e classificados no eixo "foco da prática de enfermagem" da Classificação de Fenômenos de Enfermagem da CIPE ${ }^{\circledR}$ - versão Beta cujas definições foram consideradas validadas.

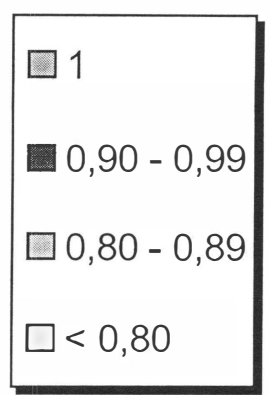

Ressalte-se que, a despeito da validação das definições conforme apresentadas no instrumento, seja na primeira ou na segunda fase, resolveu-se acatar, quando consideradas pertinentes, as sugestões dos juizes para alteração na forma de redação de algumas dessas definições.

QUADRO 1 - Listagem dos cinqüenta e quatro termos identificados no projeto CIPESC e classificados no eixo foco da prática de enfermagem da Classificação de Fenômenos de Enfermagem da CIPE ${ }^{\circledR}$ - versão Beta, cujas definições alcançaram Índice de Concordância $(\mathrm{IC})^{3} 0,80$ na primeira fase do processo de validação (número de juizes $=37$ )

continua

\begin{tabular}{|l|c|}
\hline \multicolumn{1}{|c|}{ TERMO e DEFINIÇÃO } & IC \\
\hline $\begin{array}{l}\text { Abuso de álcool: dependência física e emocional ao efeito do álcool, resultando em síndrome } \\
\text { de abstinência quando a substância é retirada (NÓBREGA; LIMA; PÉREZ, 1990). }\end{array}$ & 0,81 \\
\hline $\begin{array}{l}\text { Adoção: aceitação legal de uma pessoa como filho(a), implicando em conseqüências jurídicas } \\
\text { e psicossociais que tal situação possa ou venha a acarretar (KOOGAN/HOUAISS, 2000, } \\
\text { MICHAELIS, 1998). }\end{array}$ & 0,97 \\
\hline $\begin{array}{l}\text { Aleitamento: alimentação do recém-nascido ou lactente com leite, seja ao seio da mulher/mãe, } \\
\text { por mamadeira ou outro meio; pode ser exclusivo ou parcial, na dependência da introdução ou } \\
\text { não de outro alimento na ingestão nutricional do recém -nascido/lactente, natural, quando se } \\
\text { utiliza o leite da mama da mulher, ou artificial, quando se utiliza o leite de origem vegetal (soja) }\end{array}$ & 0,96 ou de origem animal (vaca, cabra, etc.) in natura ou industrializado (ALMEIDA, 1996). \\
\hline $\begin{array}{l}\text { Amputação: perda acidental o u excisão cirúrgica de uma parte do corpo; remoção de um } \\
\text { crescimento ou substância maligna ( ANDERSON; ANDERSON, 2001, KOOGAN/HOUAISS, } \\
\text { 2000). }\end{array}$ & 0,92 \\
\hline $\begin{array}{l}\text { Atrofia: enfraquecimento ou diminuição de volume ou de atividade fisiológica de uma parte do } \\
\text { corpo (ANDERSON; ANDERSON, 2001). }\end{array}$ & 0,97 \\
\hline $\begin{array}{l}\text { Cicatriz vacinal: marca, sinal ou vestígio na pele resultante da administração de uma vacina } \\
\text { (ANDERSON; ANDERSON, 2001). }\end{array}$ & 1,00 \\
\hline $\begin{array}{l}\text { Cidadania: principio ativo que, assegurando direitos sociais, pressupõe a necessária } \\
\text { contrapartida de dev eres ou responsabilidades (SILVEIRA, 1997); capacidade dos indivíduos } \\
\text { de participar na organização do Estado e da sociedade, contribuindo na elaboração de } \\
\text { políticas públicas capazes de concretizarem os direitos formais (BARSTED, 1994). }\end{array}$ & 0,97 \\
\hline $\begin{array}{l}\text { Comissão Local de Saúde: órgão colegiado, composto pelo Diretor e por representantes dos } \\
\text { funcionários (dos três niveis - superior, médio e elementar) da Unidade Básica de Saúde, dos } \\
\text { usuários e dos líderes da comunidade, que atua no estabelecimento de prioridades das açõ es } \\
\text { de saúde e no controle da execução da politica de saúde na instância local, inclusive nos } \\
\text { aspectos econômicos e financeiros (BRASIL, 1990). }\end{array}$ & 0,89 \\
\end{tabular}


QUADRO 1 - Listagem dos cinqüenta e quatro termos identificados no projeto CIPESC e classificados no eixo foco da prática de enfermagem da Classificação de Fenômenos de Enfermagem da CIPE ${ }^{\circledR}$ - versão Beta, cujas definições alcançaram Índice de Concordância (IC $)^{3} 0,80$ na primeira fase do processo de validação (número de juizes $=37$ )

continuação

\begin{tabular}{|c|c|}
\hline $\begin{array}{l}\text { Conselho de Saúde: órgão colegiado composto por representantes do governo, prestadores } \\
\text { de serviço, profissionais de saúde e usuários, que atua na formulação de estratégias e no } \\
\text { controle da execução da política de saúde na instância correspondente, inclusive nos aspectos } \\
\text { econômicos e financeiros (BRASIL, 1990, 1992). }\end{array}$ & 0,89 \\
\hline $\begin{array}{l}\text { Conselho Municipal de Saúde: ór gão colegiado composto por representantes do governo, } \\
\text { prestadores de serviço, profissionais de saúde e usuários, que atua na formulação de } \\
\text { estratégias e no controle da execução da política de saúde na instância municipal, inclusive } \\
\text { nos aspectos econômicose financeiros (BRASIL, 1990, 1992). }\end{array}$ & 0,89 \\
\hline $\begin{array}{l}\text { Coordenação motora: colaboração harmoniosa de partes e seqüência normal de funções } \\
\text { responsáveis pelos movimentos do corpo (MICHAELIS, 1998). }\end{array}$ & 1,00 \\
\hline $\begin{array}{l}\text { Cuidado parental: assistência do pai e da mãe, associada ao fornecimento dos aportes } \\
\text { afetivos e materiais necessários à sobrevivência e ao bem-estar dos filhos. }\end{array}$ & 0,96 \\
\hline $\begin{array}{l}\text { Deiscência: separação espontânea de partes de um órgão ou das par edes suturadas de uma } \\
\text { incisão cirúrgica; ruptura do fechamento de uma ferida (ANDERSON; ANDERSON, 2001, } \\
\text { KOOGAN/HOUAISS, 2000). }\end{array}$ & 1,00 \\
\hline Desconforto: sensação de mal-estar ou indisposição física, psicológica, espiritual ou moral. & 0,96 \\
\hline $\begin{array}{l}\text { Desenvolvimento gestacional: processo gradual de crescimento e desenvolvimento, no corpo } \\
\text { de uma mulher, de um novo ser humano, desde sua concepção até o nascimento. }\end{array}$ & 0,97 \\
\hline $\begin{array}{l}\text { Desnutrição de emoção: estado nutricional alterado, em graus variáveis, associado à ingestão } \\
\text { inadequada ou insuficiente de nutrientes, em decorrência de um desequilibrio emocional. }\end{array}$ & 0,86 \\
\hline $\begin{array}{l}\text { Destreza manual: habilidade ou aptidão para usar as mãos (KOOGAN/HOUAISS, } 2000 \text {, } \\
\text { MICHAELIS, 1998). }\end{array}$ & 0,97 \\
\hline $\begin{array}{l}\text { Empatia: capacidade de reconhecer e de compartilhar emoções e estados de espírito, bem } \\
\text { como de compreender o significado do comportamento de outra pessoa (ANDERSON; } \\
\text { ANDERSON, 2001). }\end{array}$ & 0,97 \\
\hline $\begin{array}{l}\text { Endemia: ocorrência coletiva de uma determinada doença que, no decorrer de um longo } \\
\text { periodo histórico, acometendo sistematicamente grupos humanos delimitados e } \\
\text { caracterizados, mantém a sua incidência constante, permitidas as flutuaçõ es de valores, tais } \\
\text { como as variações sazonais (BARBOSA, 1999). }\end{array}$ & 0,97 \\
\hline $\begin{array}{l}\text { Eritema: vermelhidão da pele resultante da dilatação e congestão de capilares superficiais } \\
\text { (ANDERSON; ANDERSON, 2001). }\end{array}$ & 1,00 \\
\hline $\begin{array}{l}\text { Estado vacinal: capacidade de resistência da pessoa a doenças infecciosas, conferida por } \\
\text { meio da administração de imunobiológicos. }\end{array}$ & 0,92 \\
\hline $\begin{array}{l}\text { Habilidade/capacidade motora: medida da competência ou aptidão de uma pessoa para } \\
\text { movimentar-se ou desempenhar atividades físicas. }\end{array}$ & 0,97 \\
\hline $\begin{array}{l}\text { Habilidade/capacidade sensorial: medida da competência ou aptidão de uma pessoa para } \\
\text { perceber e diferenciar sensações táteis, olfativas, gustativas, visuais e auditivas. }\end{array}$ & 0,96 \\
\hline $\begin{array}{l}\text { Higiene da gestação: conjunto de princípios e regras para evitar doenças e conservar a saúde } \\
\text { (MICHAELIS, 1998) durante o pe riodo gestacional, de modo que a gravidez seja bem } \\
\text { sucedida quanto ao bem-estar e à sobrevivência materna e da criança. }\end{array}$ & 0,97 \\
\hline $\begin{array}{l}\text { Hipercolesterolemia: quantidade de colesterol no sangue maior do que o normal (ANDERSON; } \\
\text { ANDERSON, 2001). }\end{array}$ & 0,96 \\
\hline $\begin{array}{l}\text { Hiperglicemia: qua ntidade de glicose no sangue maior do que a normal (ANDERSON; } \\
\text { ANDERSON, 2001). }\end{array}$ & 0,92 \\
\hline Hipoglicemia: quantidade $d$ & 0,92 \\
\hline
\end{tabular}


QUADRO 1 - Listagem dos cinqüenta e quatro termos identificados no projeto CIPESC e classificados no eixo foco da prática de enfermagem da Classificação de Fenômenos de Enfermagem da CIPE ${ }^{\circledR}$ - versão Beta, cujas definições alcançaram Índice de Concordância $(I C)^{3} 0,80$ na primeira fase do processo de validação (número de juízes $=37$ )

continuação

\begin{tabular}{|c|c|}
\hline $\begin{array}{l}\text { Identidade cultural: processo pelo qual os membros de um grupo ou comunidade internalizam } \\
\text { e evidencia m as caracteristicas culturais que os distinguem de outro grupo com práticas, } \\
\text { valores e crenças não semelhantes. }\end{array}$ & 0,92 \\
\hline $\begin{array}{l}\text { Incidência de câncer cérvico -uterino: número de casos novos de câncer cérvico -uterino, } \\
\text { ocorridos em uma população particular de mulheres, durante um período específico de tempo } \\
\text { (BRASIL, 1995). }\end{array}$ & 0,97 \\
\hline $\begin{array}{l}\text { Incidência de doença infecto -contagiosa: número de casos novos, ocorridos em uma } \\
\text { população particular, durante um periodo especifico de tempo, de doença causada por um } \\
\text { agente infeccioso específico ou pela toxina por ele produzida, por meio da transmissão desse } \\
\text { agente ou de seu produto tóxico, a partir de uma pessoa ou animal infectado, ou ainda de um } \\
\text { reservatório para um hospedeiro suscetivel, seja direta ou indiretamente intermediado por } \\
\text { vetor ou ambiente (BARBOSA, 1999). }\end{array}$ & 1,00 \\
\hline $\begin{array}{l}\text { Insetos nocivos: insetos capazes de provocarem danos à saúde dos seres humanos ou de } \\
\text { animais domésticos ou selvagens, por agressão e/ou por transmissão de doenças ( BRASIL, } \\
\text { 1998). }\end{array}$ & 0,97 \\
\hline $\begin{array}{l}\text { Instituição de saúde: estrutura organizacional, pública ou privada, construída e designada para } \\
\text { desenvolver atividades de diagnóstico e tratamento, visando à promoção, proteção e } \\
\text { recuperação da saúde. }\end{array}$ & 0,92 \\
\hline $\begin{array}{l}\text { Interrupção do tratamento: suspensão provisória ou permanente do plano terapêutico prescrito } \\
\text { ou recomendado para o paciente. }\end{array}$ & 0,96 \\
\hline $\begin{array}{l}\text { Laboratório de função pulmonar : conjunto de elementos destinados à realização de serviços } \\
\text { especificos necessários ao diagnóstico e à orientação terapêutica de pacientes com } \\
\text { problemas pulmonares (BRASIL, 1977). }\end{array}$ & 0,96 \\
\hline $\begin{array}{l}\text { Mama puerperal: mama de mulheres no puerpério, e em que ocorreu o processo fisiológico de } \\
\text { lactação: aumento do tecido alveolar, inicio e manutenção da secreção de leite (DERY, 1981). }\end{array}$ & 1,00 \\
\hline Manutenção do tratamento: continuidade de um plano terapêutico prescrito e iniciado. & 0,97 \\
\hline $\begin{array}{l}\text { Morbidade: comportamento de uma doença ou de um agravo à saúde em uma população } \\
\text { exposta, expressa através de coeficientes de incidência e prevalência (BARBOSA, 1999). }\end{array}$ & 0,97 \\
\hline $\begin{array}{l}\text { Mordida de animais : agressão de animais, utilizando os dentes como instrumento, podendo } \\
\text { resultar em lesão superficial ou profunda, única ou múltipla (BRASIL, 1998). }\end{array}$ & 1,00 \\
\hline $\begin{array}{l}\text { Nascido vivo : todo produto da concepção pesando no mínimo } 500 \mathrm{~g} \text { ou mais que, } \\
\text { independentemente do tempo de gestação, depois de expulso ou ex traído do corpo da mãe, } \\
\text { respira ou apresenta outro sinal de vida, tal como batimentos cardiacos, pulsação do cordão } \\
\text { umbilical ou movimentos efetivos dos músculos de contração voluntária, estando ou não } \\
\text { desprendida a placenta (BARBOSA, 1999). }\end{array}$ & 1,00 \\
\hline $\begin{array}{l}\text { Nivel de escolaridade: anos de estudo, estabelecidos em função da série e do grau mais } \\
\text { elevado alcançado pela pessoa, considerando a última série concluida com aprovação (IBGE, } \\
\text { 2001). }\end{array}$ & 1,00 \\
\hline $\begin{array}{l}\text { Paraplegia: perda de sensação, movimento e reflexos nos membros inferiores , resultante de } \\
\text { lesões traumáticas ou não traumáticas; dependendo do nivel da lesão e se o dano à medula } \\
\text { espinhal é completo ou incompleto, pode -se perder o controle intestinal e da bexiga e } \\
\text { desenvolver disfunções sexuais (ANDERSON; ANDERSON, 2001). }\end{array}$ & 0,97 \\
\hline $\begin{array}{l}\text { Prevalência de câncer cérvico -uterino: número total de casos novos e antigos de câncer } \\
\text { cérvico-uterino, ocorridos em um determinado período de tempo, usualmente expresso em } \\
\text { casos por cem mil (BRASIL, 1995). }\end{array}$ & 0,96 \\
\hline $\begin{array}{l}\text { Saneamento ambiental : conjunto de medidas a dotadas para preservar ou modificar as } \\
\text { condições do meio ambiente, com a finalidade de prevenir doenças e promover a saúde } \\
\text { (BARBOSA, 1999). }\end{array}$ & 1,00 \\
\hline
\end{tabular}


QUADRO 1 - Listagem dos cinqüenta e quatro termos identificados no projeto CIPESC e classificados no eixo foco da prática de enfermagem da Classificação de Fenômenos de Enfermagem da CIPE ${ }^{\circledR}$ - versão Beta, cujas definições alcançaram Índice de Concordância $(I C)^{3} 0,80$ na primeira fase do processo de validação (número de juizes $=37$ )

conclusão

\begin{tabular}{|c|c|}
\hline $\begin{array}{l}\text { Saneamento básico: conjunto de medidas visando a melhoria das condições de bemestar da } \\
\text { população, prevenindo doenças e promovendo a saúde através do abastecimento de água, } \\
\text { afastamento dos dejetos e remoção dos resíduos sólidos e esgoto sanitário (MOTA, 1999). }\end{array}$ & 0,97 \\
\hline $\begin{array}{l}\text { Saneamento domiciliar: conjunto de medidas adotadas para preservar ou modificar as } \\
\text { condições do domicílio, com a finalidade de prevenir doenças e promover a saúde } \\
\text { (BARBOSA, 1999). }\end{array}$ & 1,00 \\
\hline $\begin{array}{l}\text { Saúde reprodutiva: estado de completo bem-estar físico, mental e social, e não de mera } \\
\text { ausência de doença, em todos os aspectos relacionados ao sistema reprodutivo e a suas } \\
\text { funções e processos, implicando a capacidade de desfrutar de uma vida sexual satisfatória e } \\
\text { sem riscos, de procriar ou de escolher entre fazêlo ou não, no periodo e na freqüência } \\
\text { desejada (GALVÃO, 1999). }\end{array}$ & 0,84 \\
\hline $\begin{array}{l}\text { Saúde sexual: habilidade de mulheres e homens para desfrutar e expressar sua sexualidade, } \\
\text { sem riscos de doenças sexualmente transmissíveis, gestações não desejadas, coerção, } \\
\text { violência e discriminação, possibilitando-lhes experimentar uma vida sexual inform ada, } \\
\text { agradável e segura, baseada na auto-estima (GALVÃO, 1999). }\end{array}$ & 0,84 \\
\hline $\begin{array}{l}\text { Sedentarismo: condição de inatividade, como o trabalho ou recreação que se pode realizar } \\
\text { em posição sentada; padrão de vida diária que requer uma quantidade minima de esforço } \\
\text { fisico ou pouca atividade (ANDERSON; ANDERSON, 2001). }\end{array}$ & 0,97 \\
\hline $\begin{array}{l}\text { Sistema de saúde: conjunto de ações e serviços de saúde, prestados por órgãos e } \\
\text { instituições públicas federais, estaduais e municipais, da administração direta e indireta e das } \\
\text { fundações mantidas pelo Poder Público, com possibilidade de participação da iniciativa } \\
\text { privada, em caráter complementar (BRASIL, 1990). }\end{array}$ & 0,96 \\
\hline $\begin{array}{l}\text { Surto epidêmico: epidemia de proporções reduzidas, atingindo uma comunidade humana } \\
\text { (BARBOSA, 1999, BRASIL, 1977). }\end{array}$ & 0,92 \\
\hline $\begin{array}{l}\text { Taxa de evasão escolar: medida de freqüência de alunos que abandonam a escola em um } \\
\text { determinado período (IBGE, 2001). }\end{array}$ & 1,00 \\
\hline $\begin{array}{l}\text { Taxa de reprovação escolar: medida de freqüência de alunos que são reprovados em um } \\
\text { determinado ano (IBGE, 2001). }\end{array}$ & 0,97 \\
\hline $\begin{array}{l}\text { Unidade Móvel (ambulância tipo B): veículo destinado ao transporte de pacientes de alto } \\
\text { risco de vida, às emergências pré-hospitalares e ao transporte inter-hospitalar (CFM, 1998, } \\
\text { BRASIL, 1999). }\end{array}$ & 0,97 \\
\hline $\begin{array}{l}\text { Violência doméstica: variadas formas de violência interpessoal (agressão física, abuso } \\
\text { sexual, abuso psicológico e negligência) que ocorrem dentro da familia, sendo perpetradas } \\
\text { por um agressor que, em geral, possui laços de parentesco, familiares ou conjugais, e está } \\
\text { em condições de superioridade física, etária, social, psiquica e/ou hierárquica (DESLANDES; } \\
\text { GOMES; SILVA, 2000). }\end{array}$ & 0,96 \\
\hline
\end{tabular}


QUADRO 2 - Lista dos quarenta e cinco termos identificados no projeto CIPESC e classificados no eixo foco da prática de enfermagem da Classificação de Fenômenos de Enfermagem da CIPE ${ }^{\circledR}$ - versão Beta, cuja definição alcançou Índice de ConcordÂncia $(I C)^{3} 0,80$ na segunda fase do processo de validação (número de juizes $=17$ )

continua

\begin{tabular}{|c|c|}
\hline TERMO e DEFINIÇÃO & IC \\
\hline $\begin{array}{l}\text { Abandono do tratamento: quebra definitiva na continuidade, por um cliente ou familia, do plano } \\
\text { terapêutico prescrito e iniciado. }\end{array}$ & 0,94 \\
\hline $\begin{array}{l}\text { Abrigo: equipamento socia I designado ao atendimento, acolhimento e proteção de pessoas } \\
\text { (menores, idosos, migrantes, entre outros) em situação permanente ou temporária de não ter } \\
\text { espaço físico para abrigar-se. }\end{array}$ & 0,88 \\
\hline $\begin{array}{l}\text { Adolescência: período do ciclo vital do ser humano, em que se passa do ponto de } \\
\text { aparecimento inicial dos caracteres sexuais secundários para a maturidade sexual; os } \\
\text { processos psicológicos e os padrões de identificação evoluem da fase infantil para a adulta; } \\
\text { ocorre uma transição do estado de total ou parcial dependência sócio-econômica para o } \\
\text { estado de relativa independência (WHO, 1975). }\end{array}$ & 0,88 \\
\hline $\begin{array}{l}\text { Amamentação materna: aleitamento proporcionado pessoal e diretamente pela mãe, através } \\
\text { da sucção de suas mamas pelo recém-nascido/lactente. }\end{array}$ & 0,94 \\
\hline $\begin{array}{l}\text { Ambulância (tipo A): veículo destina do ao transporte de pessoas que não apresentam risco de } \\
\text { vida, para remoções simples e de caráter eletivo (CFM, 1998, BRASIL, 1999). }\end{array}$ & 1,00 \\
\hline $\begin{array}{l}\text { Ambulatório: conjunto de equipamentos e de serviços que possibilitam a prestação de } \\
\text { assistência a pessoas, sem regime de internação (BRASIL, 1983). }\end{array}$ & 0,94 \\
\hline $\begin{array}{l}\text { Animal nocivo: animal capaz de provocar riscos e danos à saúde das pessoas ou de outros } \\
\text { animais, seja por agressão e/ou por transmissão de doenças (BRASIL, 1998). }\end{array}$ & 1,00 \\
\hline $\begin{array}{l}\text { Autonomia: capacidade de autodeterminação para pens ar, querer, sentir e agir com liberdade } \\
\text { e responsabilidade (CHAUI, 1984); articula -se ao conceito de cidadania e opõe -se aos de } \\
\text { constrangimento e autoridade. }\end{array}$ & 1,00 \\
\hline $\begin{array}{l}\text { Autoproteção: conjunto de disposições, cuidados ou esforços levados a efeito para assegurar } \\
\text { a manutenção da integridade fisica, psicológica ou moral, ou para proteger os interesses, } \\
\text { sejam os da própria pessoa, da familia ou da comunidade (KOOGAN/HOUAISS, 2000, } \\
\text { MICHAELIS, 1998). }\end{array}$ & 1,00 \\
\hline $\begin{array}{l}\text { Climatério: periodo transitório entre a capacidade reprodutiva e não reprodutiva da mulher, que } \\
\text { acarreta, em resposta à interrupção da atividade gonádica, mudanças graduais, fisiológicas, } \\
\text { psicológicas e sociais (GELEIN; HEIPLE, 1981, JEFFCOATE, 1979, MACPHERSON, 1994). }\end{array}$ & 1,00 \\
\hline $\begin{array}{l}\text { Conflito: oposição entre dois sentimentos o u necessidades, simultâneos mas incompatíveis } \\
\text { (LEWIS, 2000). }\end{array}$ & 0,94 \\
\hline $\begin{array}{l}\text { Constrangimento: força ou violência fisica, psicológica ou moral exercida sobre a pessoa, } \\
\text { familia ou comunidade, para compeli -la a pensar, querer, sentir ou agir contrariamente à sua } \\
\text { vontade ou liberdade (KOOGAN/HOUAISS, 2000, MICHAELIS, 1998). }\end{array}$ & 0,94 \\
\hline $\begin{array}{l}\text { Creche: instituição que abriga, cuida e educa crianças menores de } 6 \text { anos, durante um ou dois } \\
\text { periodos do dia. }\end{array}$ & 0,82 \\
\hline $\begin{array}{l}\text { Desenvolvimento psiquico: aquele que ocorre de acordo com os costumes, valores } \\
\text { conhecimentos e habilidades do grupo social de referência, e que se realiza na relação com } \\
\text { outras pessoas (BRASIL, 1994). }\end{array}$ & 0,94 \\
\hline $\begin{array}{l}\text { Desmame: suspensão gradual ou repentina do leite materno, com introdução de outros tipos } \\
\text { de alimentos na dieta da criança (BRASIL, 1986). }\end{array}$ & 0,94 \\
\hline $\begin{array}{l}\text { Desnutrição: estado nutricional alterado, em graus variáveis, associado à ingestão } \\
\text { inadequada, não balanceada ou insuficiente de nutrientes, ou à sua assimilação ou utilização } \\
\text { reduzida pelo organismo, podendo resultar em várias conseqüênc ias biopsicossociais para a } \\
\text { pessoa (ANDERSON; ANDERSON, 2001, KOOGAN/HOUAISS, 2000). }\end{array}$ & 0,94 \\
\hline
\end{tabular}


QUADRO 2 - Lista dos quarenta e cinco termos identificados no projeto CIPESC e classificados no eixo foco da prática de enfermagem da Classificação de Fenômenos de Enfermagem da CIPE ${ }^{\circledR}$ - versão Beta, cuja definição alcançou Índice de ConcordÂncia $(I C)^{3} 0,80$ na segunda fase do processo de validação (número de juízes $=17$ )

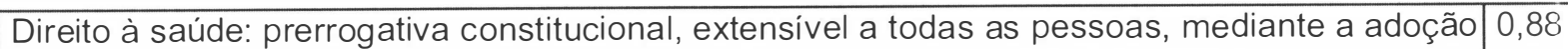
pelo Estado de politicas sociais e econômicas que visem a preservação, promoção, proteção e recuperação da saúde e a melhoria da qualidade de vida.

Direitos da clientela: prerrogativas legais de acesso universal, igualitário e eqüitativo a ações e
1,00 serviços de qualidade, para preservação, promoção, proteção e recuperação da s (BRASIL, 1990).

Epidemia: manifestação, em uma coletividade ou região, de um número de casos de alguma enfermidade ou agravo, que excede claramente a incidência prevista (BARBOSA, 1999).

$\begin{array}{ll}\text { Familia nuclear: grupo constituido por homem, mulher e filhos menores ou solteiros, vivendo } & 0,82\end{array}$ sob o mesmo teto (MICHAELIS, 1998).

Farmácia: conjunto de elementos destinado à recepção, guarda, manipulação, controle, distribuição e dispensação de drogas, medicamentos, insumos farmacêuticos e correlatos (BRASIL, 1983).

Higiene: conjunto de princípios, regras e procedimentos relacionados ao meio ambiente e às pessoas, para evitar doenças ou agravos e conservar a saúde (MICHAELIS, 1998).

Hospital: instituição designada para proporcionar à populaçã $\quad 0$, através de equipe multidisciplinar, assistência integral à saúde, usualmente em regime de internação, podendo constituir-se também em centro de educação, capacitação de recursos humanos e de pesquisas em saúde (BRASIL, 1977).

Incidência de doença de interesse escolar: número de casos novos de doença que, embora não seja considerada de notificação compulsória pela Vigilância Epidemiológica, é de interesse de notificação para bloquear surtos na escola, durante um período especifico de tempo (Ex.: parotidite, pediculose, escabiose, entre outras).

Laboratório: conjunto de elementos destinados à realização de análises clínicas necessárias ao diagnóstico e à orientação terapêutica de pacientes, ou que contribui para a descoberta, identificação e controle de agentes etiológicos de doenças ou de agravos à saúde.

Liderança: Habilidade ou capacidade, passivel de ser aprendida por indivíduos, grupos ou comunidades, com a qual se busca dirigir esforços para o comprometimento e realização de metas e objetivos comuns (ROZENDO, 2000).

Negligência: condição em que existe desatenção, descaso, ausência de diligência ou de responsabilização de uma pessoa em relação a outra, dependente de seus cuidados. Odor: impressão produzida no olfato pelas emanações voláteis (FERREIRA, 1986).

Pediculose: infestação do couro cabeludo ou de outras partes do corpo em que há concentração de pelo, por piolhos e lêndeas (ANDERSON; ANDERSON, 2001).

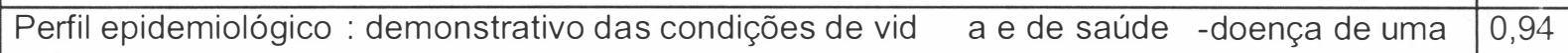
determinada população (BARBOSA, 1999), em periodos de tempo definidos.

Plano Estadual/Municipal de Saúde : conjunto articulado de proposições, metas, objetivos e estratégias para a área de saúde, a serem cumpridos durante um específico. periodo de tempo

Planta medicinal : planta cujo princípio ativo pode ser utilizado para a manutenção ou recuperação da saúde, ou para a prevenção de doenças ou de agravos à saúde.

Política de saúde: política pública, gerida pelo Est ado e dirigida à sociedade (BIREME, 2001),
0,88 resultante do embate entre os planos do aparelho ideológico do Estado e as necessidades e reivindicações de usuários e grupos organizados, para definição de metas e objetivos do sistema de saúde.

Privacidade: direito da pessoa ou de um grupo definido de pessoas à intimidade, que implica um grau de responsabilidade, culturalmente específico, de uma pessoa para com os outros, no sentido de regular o comportamento considerado de intrusão (ANDERSON; ANDERSON, 2001). 
QUADRO 2 - Lista dos quarenta e cinco termos identificados no projeto CIPESC e classificados no eixo foco da prática de enfermagem da Classificação de Fenômenos de Enfermagem da CIPE ${ }^{\circledR}$ - versão Beta, cuja definição alcançou Índice de ConcordÂncia $(\mathrm{IC})^{3} 0,80$ na segunda fase do processo de validação (número de juízes $=17$ )

conclusão

\begin{tabular}{|c|c|}
\hline $\begin{array}{l}\text { Programas Especiais de Saúde Pública: conjunto de recursos humanos, materiais e } \\
\text { financeiros e de atividades visando alcançar objetivos, definidos em politicas de saúde, } \\
\text { voltados para a promoção, manutenção e recuperação da saúde ou para a prevenção de } \\
\text { doenças ou agravos, operacionalizados localmente em função de critérios populacionais de } \\
\text { morbi-mortalidade. Exemplos: programas de controle de tuberculose, de controle de } \\
\text { hipertensão, de controle de diabetes, de saúde da criança, de saúde da mulher, entre outros } \\
\text { (BARBOSA, 1999). }\end{array}$ & 1,00 \\
\hline $\begin{array}{l}\text { Pronto Socorro: conjunto de recursos humanos, materiais e financeiros e de atividades } \\
\text { destinados à assistência de pessoas cujos agravos à saúde, com ou sem risco de vida, } \\
\text { necessitam de atenção imediata (BRASIL, 1983). }\end{array}$ & 0,82 \\
\hline $\begin{array}{l}\text { Prostituição: prática de relações sexuais ou de exposição do corpo em troca de remune } \\
\text { ou de favores (BIREME, 2001). }\end{array}$ & 1,00 \\
\hline $\begin{array}{l}\text { Qualidade de vida: percepção do individuo acerca de sua posição na vida, no contexto da } \\
\text { cultura e do sistema de valores em que vive e em relação a seus objetivos, expectativas, } \\
\text { padrões e preocupações (WHOQOL GROUP, 1995, citado por MINAYO et al., 2000). }\end{array}$ & 0,82 \\
\hline Reação vacinal: manifestação do organismo após a inoculação de determinada vacina. & 0,94 \\
\hline $\begin{array}{l}\text { Recusa para amamentar: decisão deliberada de uma mulher de não proporcionar aleitamento } \\
\text { ao recém-nascido ou lactente através da sucção de suas mamas por ele. }\end{array}$ & 0,94 \\
\hline $\begin{array}{l}\text { Recusa/rejeição: decisão deliberada de uma pessoa, família ou comunidade de não realizar, } \\
\text { no todo ou em parte, as atividades previstas no plano terapêutico prescrito ou recomendado } \\
\text { (COMPLIANCE, 1999). }\end{array}$ & 0,94 \\
\hline $\begin{array}{l}\text { Unidade Básica: estabelecimento destinado à produção e oferta de serviços de caráter } \\
\text { ambulatorial para preservação, promoção, proteção e recuperação da saúde a uma população } \\
\text { adscrita; constitui a porta de entrada do sistema de saúde. }\end{array}$ & 0,94 \\
\hline $\begin{array}{l}\text { Unidade de Exames Complementares: unidade onde são realizadas atividades de apoio } \\
\text { diagnóstico (BRASIL, 1983). }\end{array}$ & 1,00 \\
\hline $\begin{array}{l}\text { Unidade Distrital: estabelecimento destinado à produção e oferta de serviços de saúde de } \\
\text { maior complexidade; coordena e serve de referência assistencial e/ou administrativa, para } \\
\text { suprimento e atendimento das unidades de saúde, de sua área de abrangência. }\end{array}$ & 1,00 \\
\hline $\begin{array}{l}\text { Violência de rua: variadas formas de violência interpessoal (agressão física, hostilidade, roubo, } \\
\text { entre outras), perpetradas por um agressor ou conjunto de agressores a pessoas ou conjunto } \\
\text { de pessoas que transitam na rua. }\end{array}$ & 0,94 \\
\hline
\end{tabular}

A definição do termo "prevalência de doenças" não foi validada. Na primeira fase, a definição do termo como "número de casos clínicos ou de portadores de uma doença especifica, existentes em um determinado momento, em uma comunidade" alcançou um $I C=0,68$. Houve a sugestão de que essa definição fosse alterada para "número de casos clínicos, eventos ou problemas, novos e antigos, existentes em um determinado momento, em uma comunidade". Por um erro de avaliação, a sugestão não foi acatada, incluindo-se, no instrumento da segunda fase o termo "prevalência" (sem o complemento de doenças) com a seguinte definição "força com que subsistem as doenças ou agravos à saúde, novos ou antigos, em periodos de tempo definidos, em uma comunidade“. Essa definição alcançou um IC =0,71 e, dessa forma, o termo "prevalência de doenças" necessita de ser submetido a novo processo de validação.

\section{CONSIDERAÇÕES FINAIS}

Os diversos sistemas de classificação dos elementos da prática de enfermagem (diagnósticos, intervenções e resultados de enfermagem) disponiveis, sem sombra de dúvida, têm contribuido para facilitar o uso dos conhecimentos específicos da Enfermagem, para promover a autonomia profissional no julgamento sobre as necessidades de cuidado do cliente e para a realização de estudos sobre a qualidade desse cuidado. A CIPE ${ }^{\circledR}$, por exemplo, tem redundado em um avanço considerável no que diz respeito à 
intersubjetivo do significado dos termos identificados no projeto CIPESC e classificados no eixo "foco da prática de enfermagem" da Classificação de Fenômenos de Enfermagem da CIPE ${ }^{\circledR}$ - versão Beta, um aspecto fundamental a ser considerado, pois eleva seu potencial de aplicabilidade, seja no ensino, na pesquisa ou na assistência de enfermagem.

\section{REFERÊNCIAS BIBLIOGRÁFICAS:}

ALMEIDA, N. M. de. Dicionário de questões vernáculas. 3.ed. São Paulo: Ática, 1996.

ANDERSON, K. N.; ANDERSON, L. E. (Ed.) Mosby Dicionário de Enfermagem. 2.ed. São Paulo: Roca, 2001.

ASSOCIAÇÃO BRASILEIRA DE ENFERMAGEM (ABEn). Projeto de Classificação da Prática de Enfermagem em Saúde Coletiva no Brasil. Brasilia: ABEn, 1996. 22p.

BARBOSA, L. M. M. Glossário de epidemiologia \& saúde. In: ROUQUAYROL, M. Z.; ALMEIDA FILHO, N. de. Epidemiologia e saúde. 5.ed. Rio de Janeiro: MEDSI, 1999.

BARSTED, L. DE A. L. Em busca do tempo perdido: mulher e politicas públicas no Brasil 1983-1993. Estudos Feministas, CIEC/ECO/UFRJ, p.38-54, 1994. Edição Especial.

BATISTA, C. G.; MATOS, M. A. O acordo entre observadores em situação de registro cursivo: definições e medidas. Psicologia, v. 10, n. 3, p. 57-69, 1984.

BIBLIOTECA REGIONAL DE MEDICINA (BIREME). Descritores em Ciências da Saúde. Disponivel em: <http://www.bireme.br7cgi-bin/wxis1660...> Acesso em: $14 / 03 / 01$

BRASIL. Ministério da Saúde. Portaria Ministerial $\mathbf{n}^{\circ}$ $824 / 99$ - normatiza a atividade médica na área da urgência-emergência na sua fase pré-hospitalar. Brasilia, 1999.

BRASIL. Ministério da Saúde/Fundação Nacional de Saúde. Guia de Vigilßncia Epidemiológica. 5. ed. ver. ampl. Brasilia: Fundação Nacional de Saúde, 1998.

BRASIL. Ministério da Saúde. Conselho Nacional de Saúde. Comissão Nacional de Ética em Pesquisa CONEP. Resolução n. ${ }^{\circ} 196 / 96$ sobre pesquisa envolvendo seres humanos. Brasilia, 1996.

BRASIL. Ministério da Saúde. Secretaria Nacional de Assistência à Saúde. Instituto Nacional de C®ncer. Coordenadoria de Programas de Controle do C®ncer Pro-Onco. Ações de enfermagem para o controle do c®ncer. Rio de Janeiro: Pro-Onco, 1995.
BRASIL. Ministério da Saúde. Fundação Nacional de Saúde. Ações básicas de saúde e desenvolvimento da criança. Texto de apoio ao trabalho do Instrutor Supervisor na capacitação do agente comunitário de saúde. Brasilia, 1994.

BRASIL. Conselho Nacional de Saúde. Resolução $n^{\circ}$ 33/92 - aprova o documento "Recomendações para a constituição e estruturação de Conselhos Estaduais e Municipais de Saúde. Disponivel em <http// www.saude.gov.br/...> Acesso em : 25/01/01.

BRASIL. Lei n 8.080/90 - dispõe sobre as condições para a promoção, proteção e recuperação da saúde, a organização e o funcionamento dos serviços correspondentes e dá outras providências. In: CONSELHO NACIONAL DE SECRETÁRIOS MUNICIPAIS DE SAÚDE (CONASEMS). Sistema Único de Saúde. Porto Alegre: CONASEMS, dezembro 1990. Publicações Técnicas $n^{\circ} 2$.

BRASIL. Ministério da Saúde/SNPES/DINSAMI. Aleitamento materno e orientação alimentar para o desmame. 3.ed. Brasilia, 1986.

BRASIL. Ministério da Saúde/SNABS/DNOSS. Normas e padrões de construções e instalações de serviços de saúde. 2.ed. Brasilia: Centro de Documentação do Ministério da Saúde, 1983.

BRASIL. Ministério da Saúde. Portaria Ministerial $n^{\circ} 30 /$ 77 - aprova os conceitos e definições de que trata o item I do artigo $2^{\circ}$ do Decreto $n^{\circ} 76.973 / 1975$. In: BRASIL. Ministério da Saúde. Serviços de saúde: conceitos e definições. São Paulo: Edições LTR, 1977.

CHAUI, M. Participando do debate sobre mulher e violência. In: FRANCHETTO et al. (org.) Perspectivas antropológicas da mulher. Rio de Janeiro: Zahar, 1984. (Vol. 4 - Sobre mulher e violência), p.23-62.

CHIANCA, T.C.M, ANTUNES, M.J.M. (Orgs.) A classificação internacional das práticas de enfermagem em saúde coletiva: CIPESC. Brasilia: ABEN, 1999.

CLARK, J. An International Classification for Nursing Practice: Limits and perspectives. In: SIMPÓSIO INTERNACIONAL SOBRE DIAGNÓSTICO DE ENFERMAGEM, 1., 1995, São Paulo. Anais... São Paulo: Escola de Enfermagem da USP, Departamento de Enfermagem Médico-Cirúrgica, 1995. p. 34-45.

COMPLIANCE or concordance: is there a difference? Drug \& Ther Perspect, v.13, n.1, p.11-12, 1999. Disponivel em <http//www.medscape.com/adis/DTP/1999/v13.n01/ dtp1301.04/pnt-dtp1301.04.html> Acesso em 07/03/99.

CONSELHO FEDERAL DE MEDICINA (CFM). Resolução 1.529/98 - normatiza os serviços de transporte e especifica os recursos humanos e materiais necessários para seu adequado funcionamento. Brasilia: CFM, 1998. 
DERY, G. K. Lactation. In: FOGEL, C. I.; WOODS, N. F. Health care of women: a nursing perspective. St. Louis: Mosby, 1981. Chapter 25, p.547-581.

DESLANDES, S. E.; GOMES, R.; SILVA, C. M. F. P. Caracterização dos casos de violência doméstica contra a mulher atendidos em dois hospitais públicos do Rio de Janeiro. Cad. Saúde Pública, Rio de Janeiro, v.16, n.1, p.129-137, jan./mar. 2000.

EGRY, E.; MISHIMA, S.; ANTUNES, M. J. M. Classificação das práticas de enfermagem em saúde coletiva: a experiência brasileira. In: CHIANCA, T.C.M , ANTUNES, M.J.M. (Orgs.) A classificação internacional das práticas de enfermagem em saúde coletiva. CIPESC. Brasilia: ABEN, 1999.

FERREIRA, A. B. H. Dicionário da língua portuguesa. Rio de Janeiro: Nova fronteira, 1986.

GALVÃO, L. Saúde sexual e reprodutiva, saúde da mulher e saúde materna: a evolução dos conceitos no mundo e no Brasil. In: GALVÃO, L.; DÍAZ, J. Saúde sexual e reprodutiva no Brasil: dilemas e desafios. São Paulo: HUCITEC/Population Council, 1999. Cap.5.

GARCIA, T. R.; NÓBREGA, M. M. L. (Orgs.). Sistemas de Classificação em Enfermagem: um trabalho coletivo. João Pessoa: ABEn, 2000a. (Série Didática: Enfermagem no SUS).

GARCIA, T. R.; NÓBREGA, M. M. L. Inventário Vocabular de Fenômenos e Ações de Enfermagem em Saúde Coletiva. In: GARCIA, T. R.; NÓBREGA, M. M. L. (Orgs.). Sistemas de Classificação em Enfermagem: um trabalho coletivo. João Pessoa: ABEn, 2000b. (Série Didática: Enfermagem no SUS), p.83-170.

GELEIN, J. L.; HEIPLE, P. Aging. In: FOGEL, C. I.; WOODS, N. F. Health care of women: a nursing perspective. St. Louis: Mosby, 1981. Chapter 18, p.363-394.

INSTITUTO BRASILEIRO DE GEOGRAFIA E ESTATISTICA (IBGE). Indicadores sociais mínimos: conceitos. Disponivel em: <http://www.ibge.net/ibge/esta...odevida/ indicadoresminimos/ conceito.shtm> Acesso em 20 mar. 2001.
INTERNATIONAL COUNCIL OF NURSES (ICN). Proyecto ICNP® para los paises financiado por la Fundación $W$. K. Kellogg. Disponivel em: <http://www.icn.ch/ icnpkelloggsp.htm> Acesso em 27 jun. 2000.

JefFCOATE, N. Princípios de ginecologia. 4.ed. São Paulo: Manole, 1979.

KOOGAN/HOUAISS, enciclopédia e dicionário ilustrado. [direção geral, Abrahão Koogan; supervisão editorial, Antônio Houaiss]. 4.ed. Rio de Janeiro: Seifer, 2000.

LEWIS, A. Wordweb. 2000. Disponivel em: <http:// www.wordweb.co.uk>.

MacPHERSON, K. †. Menopause as disease: the social construction of a metaphor. In: CHINN, P. L. (Ed.) Developing nursing perspectives in women's health. Gaithersburg: Aspen Publishers, 1994. p.48-66

MICHAELIS: moderno dicionário da língua portuguesa. São Paulo: Companhia Melhoramentos, 1998.

MINAYO et al. Qualidade de vida e saúde: um debate necessário. Ciência \& Saúde Coletiva, v.5, n.1, p.7-18, 2000.

MOTA, S. Saneamento. In: ROUQUAYROL, M. Z.; ALMEIDA FILHO, N. de. Epidemiologia e saúde. 5.ed. Rio de Janeiro: MEDSI, 1999.

NÓBREGA, M. M. L.; LIMA, M. D. C.; PÉREZ, V. L. A. B. Glossário de termos psiquiátricos. João Pessoa: Almeida, 1990

ROZENDO, C. A. A liderança no cotidiano da enfermagem hospitalar: entre luzes e sombras. Ribeirão Preto, 2000. 229 f. Tese (Doutorado) - Escola de Enfermagem de Ribeirão Preto - USP:

SILVEIRA, R. M. G. A cidadania no atual processo político brasileiro. Revista ADUFPB-JP, n.2, p.46-57, out. 1997.

WORLD HEALTH ORGANIZATION (WHO). Pregnancy and abortion in adolescence. Geneva: WHO, 1975. (Technical Report Series, n.583). 